\title{
Feline Panleukopenia Infections : Treatment and Control in Nepal
}

\author{
Shankar Pandey
}

\section{ABSTRACT}

Feline panleukopenia is a fatal and contagious viral disease caused by the family of Parvoviridae. Feline panleukopenia virus (FPV) is synonymously called as feline distemper and feline Parvo. FPV infects all felids, raccoons, mink and foxes. FPV infected animals generally have compromised health conditions. Cats of all ages are mostly affected by FPV, but kittens are highly susceptible with higher mortality. The virus is resistant to many disinfectants and normally survives in the environment for several months. Transmission occurs through direct contact of faeces contaminated feeds as well as by indirect contact of fomites, and through intrauterine route. Clinical symptoms include vomition, diarrhoea, lymphopenia and neutropenia, followed by thrombocytopenia and anaemia, immunosuppression, cerebellar ataxia and abortion. Commercially available test kits are used to detect FPV antigen in faeces. Fluid therapy, antibiotics, metronidazole, supportive therapy, and good nursing care are the common treatment practice in Central Referral Veterinary Hospital (CRVH), Nepal. The disease can be controlled by isolation of infected cats, routine vaccination and disinfection of premises. The higher incidence in Nepal is due to lack of vaccination practice against FPV. Disinfectants containing sodium hypochlorite (bleach), potassium peroxymonosulfate and accelerated hydrogen peroxide are found effective. The aim of this paper is to review the current status of the disease, epidemiology, pathophysiology, diagnosis, treatment and control measures for prevention and management of feline panleukopenia infections in developing countries like Nepal.

Keywords: Disinfectants, feline distemper, FPV, vaccination.

Published Online: February 18, 2022

ISSN: 2736-6596

DOI : 10.24018/ejvetmed.2022.2.1.19

S. Pandey*

Central Referral Veterinary Hospital,

Tripureshwor, Kathmandu, Nepal.

(e-mail: drspvet@gmail.com)

*Corresponding Author

\section{INTRODUCTION}

Feline panleukopenia virus (FPV) is a fatal, highly contagious, viral infectious disease of cats caused by the feline parvovirus with the peculiar hematological alteration of leukopenia thus known as feline panleukopenia. The disease is popularly called feline distemper or feline parvo interchangeably. Though the names are similar, feline distemper and feline parvovirus (FPV) should not be confused with canine distemper (CD) or canine parvovirus $(\mathrm{CPV})$, the reason behind this is that they are caused by different viruses.

FPV is vaccine-preventable disease as it is mostly seen in unvaccinated cats and kittens. Kittens are most susceptible and severely affected by the virus. FPV can infect all species of Felidae as well as raccoon, ferrets, and mink [1]. The viruses do not infect people. The feline parvovirus infects and kills cells that are associated with replication in and cytopathic effects of rapidly growing and dividing, such as those in the bone marrow, lymphoid cells, intestines, and the developing fetus resulting in congenital abnormalities in late gestation period [2].

Feline panleukopenia is caused by a small, single-stranded deoxyribonucleic acid (DNA) virus and is closely related to
CPV. Cats with feline panleukopenia virus infection may also be infected with CPV strains (namely 2a, 2b, and 2c). Cats start to shed virus a few days after infection up to 6 weeks. The causative FPV virus is very resistant; and at room temperature, they can survive for a year in the presence of organic matters. Viral persistence in the environment plays an important role in disease transmission from shelters, farms, and urban stray populations [3], [4].

\section{MATERIALS AND METHODS}

The study is performed by systematic review of FPV infections in cats in various literatures and by incorporating the treatment practice of CRVH.

\section{DESCRIPTION OF FPV}

\section{A. Comparison of Feline Panleukopenia Virus (FPV) with Canine Parvovirus (CPV)}

Historically, CPV has been evolved from FPV in 1970s. Both FPV and CPV are closely related and antigenically stable since their DNAs share about $80 \%$ of the restriction sites tested [5], [6]. An average incubation period of both of 
the diseases ranges between four to seven days, but can extend up to two weeks in some animals. Moreover, feline panleukopenia virus (FPV) is closely related to the canine parvovirus-2 (CPV-2) and its antigenic variants CPV-2a, CPV-2b, and CPV-2c. Several studies suggested that FPV is the ancestral origin of CPV-2 [7], [8].

\section{B. Epidemiology and Risk Factors of FPV}

The virus has been distributed worldwide irrespective of geography, climate and temperature. Common reservoirs of FPV are kennels, pet shops, stray cats, animal shelters, and unvaccinated feline populations. Urban areas have dense stray cat populations which appear to have more outbreaks in warm season through direct contact [9]. Virtually all kittens and cats are infected by the virus at some point in their lives as the virus is present everywhere. Cats of any age may be infected with FPV; however, young, debilitated, and unvaccinated cats are found mostly susceptible. Death from FPV is more common at the age of 3-5 months [4], [9]. Fecaloral transmission is predominant but it also spread through other secretions and urine. FPV virus is a highly contagious and stubbornly persistent in the environment in densely populated area with variable vaccination status [10].

\section{Transmission and Pathophysiology}

Transmission is through direct contact with infected cats or via fomites (bedding, food dishes); fleas and humans. Infected cats shed enormous numbers of FPV virions in the feces, vomitus, urine, nasal secretions, and saliva [11]; which are found active up to 6 weeks after the onset of clinical signs. Since the virus survives for few days up to a year in the environment, cats may be infected from the environment even without direct contact with an infected cat [12]. When susceptible cats come in contact with these secretions, or even the insects or fleas from infected cats, infection occurs. Other sources of infection are bedding, cages, food bins, and contaminated persons. Therefore, infected cats should be isolated and treated accordingly. The premises with infected cats should be sanitized properly and infected materials should be destroyed or disposed with strict measures [9].

After intranasal or oral transmission, the virus initially replicates in the macrophages of the oropharynx and regional lymph nodes, and distributes throughout the body [13]. Mostly FPV infects rapidly dividing cells of intestinal epithelial cells, lymphatic tissues, and bone marrows. After clinical onset, FPV shows aggravations with secondary bacterial infection, translocation of gut bacteria, and increased susceptibility to bacterial infection [14].

\section{Clinical Findings}

Usually less than one year old cats become sick. Per-acute cases may die suddenly with little or no symptoms. Acute cases show fever $\left(104^{\circ}-107^{\circ} \mathrm{F}\right)$, depression, and anorexia after an incubation period of 2-7 days. Some cats show intermittent fever, and sometimes, the temperature abruptly falls down to lower-than-normal levels shortly before death [4]. The FP virus damages the cells that line the intestines. The effect on bone marrow and lymph nodes results in shortages of all types of white blood cells (panleukopenia) and of red blood cells (anemia). Signs and symptoms include depression, inappetence, high fever, lethargy, vomiting, severe diarrhea, nasal discharge, and dehydration.
Hypersalivation, nausea, abdominal pain and foul-smelling diarrhoea are observed in later stage. In $15 \%$ of cases, the vomit is bilious, and the diarrhea is hemorrhagic [4], [15].

Feline parvovirus (FPV) causes an acute, sometimes fatal, enteritis in cats of all ages. In symptomatic animals, FPV causes a unique diagnostic smell in faeces. Bloody enteritis, vomiting, and leucopenia can lead to secondary bacterial infection. These signs are hallmarks to identify FPV infections [16].

Clinical symptoms in newborn kittens shows high mortality $(>90 \%)$ with per-acute deaths and neurological disorders like ataxia and blindness. Older kittens develop similar symptoms of panleukopenia, neutropenia and diarrhoea which are due to infection of bone marrow, lymphatic tissue and intestinal epithelial cells. Clinical signs are most often seen in kittens of 2 to 5 months of age and older cats predominately exhibit subclinical or mild forms of the disease [17].

Pregnant cats with FPV infection may lead to abortion, stillbirths, fading kittens, or congenital defects depending on the stage of gestation. These congenital defects may include cerebellar hypoplasia, ataxia, hydranencephaly, and cardiomyopathy. Early neonatal period may show similar abnormalities and young feline may get brain and eye abnormalities as well [2], [18].

\section{Diagnosis}

Feline panleukopenia is diagnosed on the basis of: (a) history and clinical findings, (b) hematological alterations like severe leukopenia, neutropenia, anemia, and thrombocytopenia, (c) serum biochemistry analysis that shows increased hepatic enzyme activities and by (d) virus detection using commercial fecal enzyme-linked immunosorbent assay (ELISA) kits. PCR assays are also available for detection and isolation of viral DNA in fecal and tissue samples from affected cats [3]. Differential diagnosis should be done with similar illnesses such as salmonellosis, campylobacteriasis, pancreatitis, feline immunodeficiency virus infection, and feline leukemia virus infection [2], [9].

\section{A. Feline Panleukopenia Virus (FPV) Ag Rapid Test Kit}

To detect the virus in fecal samples, fecal ELISA test kits are designed to detect CPV-2 variants of dogs that also detect FPV. Most kits use monoclonal antibodies specific for a single epitope of the virus [19]. Feline Panleukopenia Virus (FPV) Ag rapid test is rapid, accurate and easy-to-operate based on sandwich immunochromatographic assay, to detect feline panleukopenia virus (FPV) antigen in feces. The steps include faecal swab collection, mixing of swab in buffer solution, putting three drops of the mixture in specimen well of the cassette and reading of result in five minutes. Results are positive if $\mathrm{T}$ and $\mathrm{C}$ is marked, negative if only $\mathrm{C}$ is marked and invalid if only $\mathrm{T}$ is marked [20]. False negatives are relatively common, but false positive parvo results are not very common. In some cases, immediately after vaccination, modified live panleukopenia vaccines may result in a positive test [21]. 


\section{Common Treatment Practice}

As FPV infection is a virus-induced situation, there is no specific treatment. Therefore, treatment should be aimed at controlling the secondary bacterial infection and providing symptomatic treatment with supportive care [22]. This includes fluid therapy to correct dehydration and electrolyte abnormalities, antibiotics to fight off secondary bacterial infections, and antiprotozoal and antidiarrheal medicines to control vomition and diarrhea. If there is significant leukopenia and the rapid parvo test is positive, the success of treatment relies on catching the disease in earlier stages and proceeding with aggressive treatment, including white blood cell stimulating medicines such as Neupogen [10]. Anticipation of these possibilities, close monitoring, and prompt intervention improve the outcome.

\section{A. Treatment of FPV at Central Referral Veterinary Hospital (CRVH)}

The FPV treatment protocol of CRVH has been described that showed successful results. Fluid Therapy: Cats require the fluids about 3 to $4.4 \%$ of body weight per day, if cat is not eating. This quantity can be reduced if feeding is possible. Isotonic crystalloid solutions (eg, Ringer's lactate (RL) or Normal saline (NS) solution) is given @ 30-40 ml per kg body weight per 24 hours for 3-5 days together with 5\% glucose if there is hypoglycemia. Sub-cutaneous (SC) fluids should be limited to $135 \mathrm{ml}$ in an adult at one time and decreased for kittens. Antibiotics: Leucocytopenia is treated with broad spectrum antibiotics immediately after diagnosis. If there is hypothermia, I/V antibiotics are preferred. Most commonly an injectable ceftriaxone injection (20-30 mg per $\mathrm{kg}$ ) or an injectable penicillin drug (penicillin $\mathrm{G}$ procaine (20,000 units per $\mathrm{kg}$ ) is given 1-2 times a day for 3-5 days. Injectable antibiotics are given if there is a lot of vomiting. Metronidazole: Metronidazole is used parenterally through I/V fluids to control protozoal diarrhoea and given @ 5-15 ml for kittens and 20-30 ml for adult cats for 3-5 days. Temperature management: Regular monitoring of body temperature is recommended and should be kept under 103$104 \mathrm{OF}$, but fever for some time may be beneficial to fight against the viral infection. Fever above $104{ }^{\circ} \mathrm{F}$ should be dropped down by using fluids or cold pack or antiinflammatory drugs such as meloxicam @ $0.2 \mathrm{mg}$ per kg. Supplements: Vitamin B-complex injections (Conciplex @ 0.1 to $0.25 \mathrm{ml}$ for kitten and up to $0.75 \mathrm{ml}$ for adult) every day for 3-5 days is extremely helpful and important. This is given along with $\mathrm{I} / \mathrm{V}$ infusions. Feeding control: In most of the cases, parenteral infusions without food has shown successful result. Therefore, minimal or no feeding principle should be adopted during the treatment of FPV. Vomiting must be controlled by using antiemetic drugs metoclopramide@ 0.1-0.15 ml for kittens and 0.3-0.5 ml for adult or by injectable ondansetron@0.1 mg per kg. Liver tonics: The excess liver enzymes causes excessive vomiting and inappetence. To boost up appetite, liver tonics with silymarin is commonly used (Hepamust @ 1-2 ml orally) for 10-15 days.

\section{B. Prognosis of FPV}

The mortality rate is directly proportional to the severity of the leukopenia. The prognosis may be worse if there is a profound leukopenia (WBC count falls below 1000 cells per $\mathrm{ml}$ of blood) [11]. Feline panleukopenia has noticeably worse prognosis than CPV enteritis. In feline panleukopenia, the negative prognostic factors are leukopenia, thrombocytopenia, hypoalbuminemia, and hypokalemia. The In-shelter environments, survival rates of $20 \%-51 \%$ have been reported in cats with supportive treatment in hospital [4], [23], [24]. In a study by [23] indicated that survival rate was $51.1 \%$ and there was no significant correlation between the outcome of treatment and living conditions, age, vaccination status, or severity of clinical signs.

\section{Control And Prevention}

Sanitation and environmental control are essential for successful FPV control. Contaminating surfaces, food bowls, litter trays and other fomites should be disinfected by best cleaning agents. The lingering effects of panleukopenia virus should not be underestimated. This is because the virus can live on surfaces for a year or more. So, it is not recommended to take any kittens into contaminated space without vaccination [10]. Quaternary ammonium compounds are found ineffective against FPV. The panleukopenia virus is killed by recommended dilute bleach of sodium hypochlorite solution (1 part bleach to 32 parts water). This solution is suitable for disinfecting food bowls, litter pans, cages, and other surfaces. Potassium peroxymonosulfate (Trifectant or Virkon) and accelerated hydrogen peroxide (Accel or Rescue) both have greater detergent properties and better activity against organic matter in comparison to bleach and related products and reliably destroys FPV [10], [12].

To prevent nosocomial infections, infected cats should be strictly isolated for 4-6 weeks either at home or hospital. This period is risky because of shedding of high quantity of virus at that duration [12].

\section{A. Vaccination for FPV}

Control through vaccination is found effective since FPV is not appeared in vaccinated domestic cats, but is problematic in feral, shelter, and stray cats [14]. Females with high levels of neutralizing antibodies will protect kittens through passive transfer. Also, the primary vaccination should be delayed until MDA (maternally derived antibodies) titres have declined because even very low titres of MDA apparently inhibit the development of active immunity [25]. FP vaccines are available in inactivated and modified live virus (MLV) forms [12], [26]. Excellent inactivated and modified-live virus vaccines provide solid, long-lasting immunity. Modified live vaccines should not be used during pregnancy or sick and immunosuppressed cats or in kittens aged less than 4 weeks [2]. This is because of the danger of development of cerebellar hypoplasia in kittens. Killed vaccine may be an option for the use in immediate exposure conditions [10].

FPV vaccination schedule starts with an initial subcutaneous dose at 6-9 weeks of age, followed by first booster injection at 3-4 weeks age and second booster injection after one year, and in every 3 years thereafter. Low levels of MDAs do not protect against FPV but impede development of a good humoral immune response to vaccination. To overcome the problem of MDAs, it is 
recommended to have a third vaccine dose 3-4 weeks after the second, given no earlier than 16 weeks of age. There is lifelong protection following natural infection of FPV. Therefore, booster vaccination beyond 12 weeks of age is essential to prevent panleukopenia [22], [27]. FPV vaccines can induce high antibody titres and protection from challenge for up to seven years [4], [12]. Regardless, manufacturers recommend more frequent immunization to guarantee continuous protection.

American Veterinary Medical Association (2010) states that previously this disease was considered as major cause of feline deaths, but nowadays, due to massive vaccination and application of control measures, it is being a preventable disease of cats. Therefore, Feline panleukopenia is considered as a vaccine-preventable disease and successful vaccination induces virus-neutralizing antibodies providing protection against clinical FPV [17], [28].

\section{CONCLUSION}

Feline panleukopenia infection pose a threat to feline health. Unvaccinated cats are highly susceptible and at risk of FVP. Environmental control measures, isolation of infected animals and due treatment should be complemented by blanket vaccination to provide protection at the population level. Vaccines are found to be highly efficacious for the protection against FPV. The high incidence of clinical cases in Nepal is due to lack of vaccination practice for FPV for adults and kittens and the expensiveness of the vaccine. Therefore, cost effective FPV vaccines should be made available for routine tailored vaccination. To our knowledge, though there are numerous cases of FPV reported in Central Referral Veterinary Hospital (CRVH), Kathmandu, Nepal, however, there is not enough documentation regarding the FPV infection in Nepal. Therefore, further study on prevalence of the FPV in countries like Nepal is highly recommended.

\section{ACKNOWLEDGMENT}

I would like to acknowledge the technical team of CRVH who assisted me during the study. Furthermore, I would like to thank my wife Sanjita Acharya for her generous support to prepare the article.

\section{REFERENCES}

[1] Sherding RG. Intestinal Viruses. Saunders Manual of Small Animal Practice. [Internet] 2021 [Cited 2021 Nov 11]; Available from: https://www.sciencedirect.com/topics/ veterinary-science-andveterinary-medicine/feline-panleukopenia.

[2] Evermann JF, Kennedy MA. Viral Infections in Small Animal Pediatrics. [Internet] 2011 [cited 2021 Dec 7]; Available from: https://www.sciencedirect.com/topics/veterinary-science-andveterinary-medicine/feline-panleukopenia.

[3] Sykes JE. Viral Infections in Small Animal Critical Care Medicine. [Internet] 2009 [cited 2021 Nov 22]; Available from: https://www.sciencedirect.com/topics/veterinary-science-andveterinary-medicine/feline-panleukopenia.

[4] Squires RA. Feline Panleukopenia, Merck Veterinary Manual. [Internet] 2020 [cited 2021 Dec 10]; Available from: https://www.msdvetmanual.com/generalized-conditions/felinepanleukopenia/feline-panleukopenia\#v3276627.
[5] Lenghaus C, Studdert MJ. Relationship of canine panleukopenia (enteritis) and myocarditis parvoviruses to feline panleukopenia virus. Australian Veterinary Journal. 1980; 56:152-153.

[6] Tratschin J, Mcmaster GK, Kronauer G, Siegl G. Canine Parvovirus: Relationship to Wild-type and Vaccine Strains of Feline Panleukopenia Virus and Mink Enteritis Virus J. Gen. Virol. 1982; 61: 3341.

[7] Shackelton LA, Parrish CR, Truyen U, Holmes EC. High rate of viral evolution associated with the emergence of carnivore parvovirus. Proc. Natl. Acad. Sci. 2005; 102: 379-384.

[8] Truyen U. Evolution of canine parvovirus-a need for new vaccines? Vet. Microbiol. 2006; 117: 9-13.

[9] AVMA. Feline Panleukopenia. [Internet] 2021 [cited 2021 December 5]; Available from: https://www.avma.org/resources-tools/petowners/petcare /feline-panleukopenia.

[10] Rice JK. Successful Treatment of Feline Panleukopenia: A Guideline for Rescuers and Veterinarians, Part I. J Vet Sci Med Diagn. 2017; 6: 2 .

[11] MacLachlan NJ, Dubovi EJ. Fenner's Veterinary Virology, Fourth Edition. 2011

[12] Dickinson M. Feline Panleukopenia: Risk Factors, Prevention and Vaccines. 2020. [Internet] 2020 [cited 2021 Nov 12]; Available from: https://www.dispomed.com/feline-panleukopenia-risk-factorsprevention-and-vaccines.

[13] Boes KM, Durham AC. Bone Marrow, Blood Cells, and the Lymphoid/Lymphatic System in Pathologic Basis of Veterinary Disease. 2017. [Internet] [cited 2021 Nov 21]; Available from: https://www.sciencedirect.com/topics/ veterinary-science-andveterinary-medicine/feline-panleukopenia.

[14] Cohn LA, Langdon P. Handbook of Small Animal Practice. 2008. [Internet] [cited 2021 Dec 11]; Available from: https://www.sciencedirect.com/topics/veterinary-science-andveterinary-medicine/feline-panleukopenia.

[15] Truyen U, Addie D, Belák S, Boucraut-Baralon C, Egberink H, Frymus, T. et al. Feline Panleukopenia: ABCD Guidelines on Prevention and Management. Journal of Feline Medicine and Surgery. 2009; 11(7): 538-546.

[16] Spindel, M. Exploring the myths, finding the facts Canine and feline parvovirus: What you need to know, [Internet] 2021 [cited 2021 Nov 27]; Available from: https://humanepro.org/magazine/articles/canineand-feline-parvovirus-what-you-need-know.

[17] Greene CE, Addie DD. Feline Parvovirus Infections. Infectious diseases of the dog and cat. 2006: 78-88.

[18] Carmichael LE, Binn LN. New enteric viruses in the dog. Advances in Veterinary Science and Comparative Medicine. 1981, 25: 1-37.

[19] Stokes J, Lappin MR. Infectious Diseases in the Cat. [Internet] 2012 [cited $2021 \quad$ Dec 8]; Available from: https://www.sciencedirect.com/topics/veterinary-science-andveterinary-medicine/feline-panleukopenia.

[20] Biopanda, Feline Panleukopenia Virus Antigen Rapid Test. [Internet] 2021 [cited 2021 Dec 7]; Available from: https://www.biopanda.co.uk/php/products/vet/feline_panleukopenia.p hp

[21] Patterson EV, Reese MJ, Tucker SJ, Dubovi EJ, Crawford PC, Levy, JK. Effect of vaccination on parvovirus antigen testing in kittens. J Am Vet Med Assoc. 2007; 230: 359-363.

[22] Simpson KW, Birnbaum N. Fluid and electrolyte disturbances in gastrointestinal and pancreatic disease. In DiBartola SP, editor, Fluid, Electrolyte and Acid-Base Disorders in Small Animal Practice, St. Louis, 2006: 420-436.

[23] Kruse BD, Unterer S, Horlacher K, Sauter-Louis C, Hartmann K. Prognostic Factors in Cats with Feline Panleukopenia. $J$ Vet Intern Med. 2010; 24: 1271-1276.

[24] Wolfesberger B, Tichy A, Affenzeller N, Galler A, Shibly S. Clinical outcome of 73 cases with feline panleukopenia. Wien Tierärztl Monat. 2012; 99: 235-241.

[25] Jakel V, Cussler K, Hanschmann KM, Truyen U, König M, Kamphuis E. et al. Vaccination against Feline Panleukopenia: implications from a field study in kittens; BMC Vet Res. 2012; 8: 62.

[26] Gaskell RM, Tennant B, Bennett M, Willoughby K. Feline and Canine Infectious Diseases. 1996.

[27] Esfandiari J, Klingeborn B. A comparative study of a new rapid and one-step test for the detection of parvovirus in faeces from dogs, cats and mink. J. Vet. Med. B Infect. Dis. Vet. Public Health. 2000; 47: 145153.

[28] Scott FW, Csiza CK, Gillespie JH. Maternally derived immunity to feline panleukopenia. J Am Vet Med Assoc. 1970; 156(4): 439-453. 


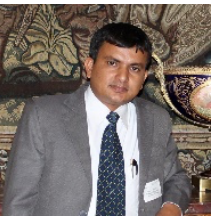

S. Pandey (Kathmandu Nepal; 23 March 1977) has achieved bachelor's degree in veterinary science and animal husbandry as well as master's degree in veterinary pharmacology from Tribhuvan University, Institute of Agriculture and Animal Science. The major field of study and work is antimicrobial drug residues in animal products, drug usage and complications, diseases of veterinary importance and their treatment.

He works in Department of Livestock Services (DLS) Nepal and now working as senior veterinary officer at Central Referral Veterinary Hospital (CRVH), Kathmandu, Nepal. He has worked for DLS for more than 15 years as civil servant. He is experienced clinician and working for ruminants and pet animals. He has publications published in various technical bulletins and journals.

Dr Pandey is a member of Nepal Veterinary Council, Nepal Veterinary Association and Veterinary Practitioner's Association of Nepal. Dr Pandey is taking leadership to implement animal birth control program for dogs and cats (Spaying and Castration) all over the country through national campaign to eliminate dog mediated rabies by 2030 .. 